\title{
On One Justification on the Use of Hybrids for the Solution of First Order Initial Value Problems of Ordinary Differential Equations
}

\author{
Kamoh Nathaniel Mahwash ${ }^{1}$, Gyemang Dauda Gyang ${ }^{2}$, Soomiyol Mrumun Comfort ${ }^{3}$ \\ ${ }^{1}$ Department of Mathematics/Statistics, Bingham University, Karu, Nigeria \\ ${ }^{2}$ Department of Mathematics and Computer Science, Benue State University, Makurdi, Nigeria \\ ${ }^{3}$ Department of Mathematics/Statistics, Plateau State Polytechnic, BarkinLadi, Nigeria
}

Email address:

mahwash1477@gmail.com (K. N. Mahwash), daudagyemang@yahoo.com (G. D. Gyang), mrumunsoomiyol@yahoo.com (S. M. Comfort)

\section{To cite this article:}

Kamoh Nathaniel Mahwash, Gyemang Dauda Gyang, Soomiyol Mrumun Comfort. On One Justification on the Use of Hybrids for the Solution of First Order Initial Value Problems of Ordinary Differential Equations. Pure and Applied Mathematics Journal. Vol. 6, No. 5, 2017, pp. 137-143. doi: 10.11648/j.pamj.20170605.11

Received: September 7, 2017; Accepted: September 18, 2017; Published: October 11, 2017

\begin{abstract}
This paper is aimed at discussing and comparing the performance of standard method with its hybrid method of the same step number for the solution of first order initial value problems of ordinary differential equations. The continuous formulation for both methods was obtained via interpolation and collocation with the application of the shifted Legendre polynomials as approximate solution which was evaluated at some selected grid points to generate the discrete block methods. The order, consistency, zero stability, convergent and stability regions for both methods were investigated. The methods were then applied in block form as simultaneous numerical integrators over non-overlapping intervals. The results revealed that the hybrid method converges faster than the standard method and has minimum absolute error values.
\end{abstract}

Keywords: Hybrid Method, Collocation, Interpolation, Shifted Legendre Polynomials Approximation, Continuous Block Method, Order, Consistency, Zero Stability, Convergent

\section{Introduction}

Most physical phenomena in science and engineering used mathematical models to help in the understanding of the physical world problems. These models often yield equations that contain some derivatives of an unknown function of one or several variables. Such equations are called differential equations. Differential equations play an important role in the modeling of physical problems arising from almost every discipline of study such as economics, medicine, psychology, operation research, space technology and even in areas such as biology and astronomy.

Interestingly, differential equations arising from the modeling of such physical phenomena often are very difficult or impossible to solve analytically. Hence, the need for the development of numerical methods to obtain approximate solutions becomes inevitable.

Many scholars have worked extensively on the solution of differential equations. These authors proposed different methods ranging from predictor corrector method to block method using different polynomials as basis functions evaluated at some desired points.

In this work, two types of block methods are proposed, the first is the five-step standard block method and the second is the five-step hybrid method with four off- grid points, using the shifted Legendre polynomials evaluated at some grid and off-grid points to give the needed discrete schemes.

Consider a numerical method for solving general first order initial value problems of ordinary differential equations of the form:

$$
y^{\prime}=f(x, y), y(0)=y_{0}
$$

where $f$ is a continuous function and satisfies Lipschitz condition of the existence and uniqueness of solution. Many authors have proposed solution for first order initial value problems of ordinary differential equations using different approaches. This work will adopt the block method technique for solving (1) as suggested by researchers. [4], considered 
the Continuous implicit hybrid one step methods for the solution of initial value problems of general second order ordinary differential equations. [5], introduced the application of two step continuous hybrid Butcher's method in block form for the solution of first order initial value problems; this approach eliminates requirements for a starting value. [2], introduced a new hybrid method for systems of stiff equations. [11], developed a new Butcher type two-step block hybrid multistep method for accurate and efficient parallel solution of ordinary differential equations. [1], used hybrid formula of order four to generate starting values for Numerov method. [3], developed linear multistep hybrid methods with continuous coefficients for solving stiff ordinary differential equations. [10], introduced a hybrid linear collocation multistep scheme for solving first order initial value problems of ordinary differential equations. [9], developed a three step implicit hybrid linear multistep method for the solution of third order ordinary differential equations.

\section{Derivation of the Method}

In this section, two methods are developed namely fivestep block method and five-step with three off-grid points, by interpolating and collocating at some selected points.

Consider the shifted Legendre approximation of the form

$$
y(x)=\sum_{i=0}^{\phi} C_{i} P_{i}(t)
$$

where $\varphi=m+s-1, m$ and $s$ are interpolation and collocation points. The first derivative of (2) gives

$$
y^{\prime}(x)=\sum_{i=0}^{\phi} C_{i} P_{i}^{\prime}(t)
$$

substituting (3) in (1), to get

$$
f(x, y)=\sum_{i=0}^{\phi} C_{i} P_{i}^{\prime}(t)
$$

\subsection{Five Step Method}

Interpolating (2) at $x_{n+4}$ and collocating (3) at $x_{n}, x_{n+1}$, $x_{n+2}, x_{n+3}, x_{n+4}$ and $x_{n+5}$ gives the system of nonlinear equations of the form

$$
\mathrm{AX}=\mathrm{B}
$$

where

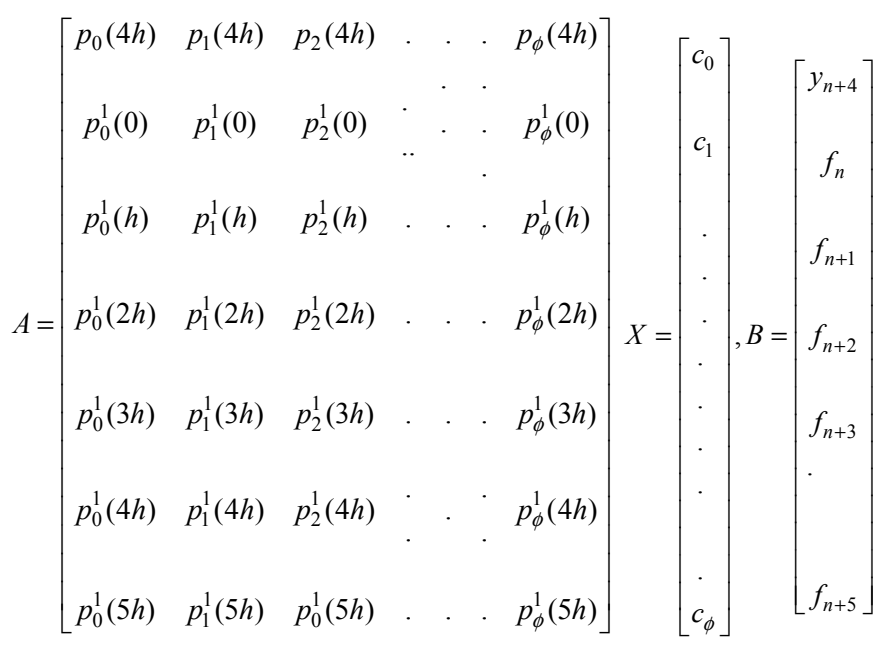

Solving for the $C_{i}$ 's using inverse of a matrix method and substituting in (2) gives the continuous formulation

$$
y(x)=\sum_{j=0}^{4} \alpha_{j}(x) y_{n+j}+h \sum_{j=0}^{5} \beta_{j}(x) f_{n+j}
$$

where

$$
\begin{gathered}
\alpha_{0}=\alpha_{1}=\alpha_{2}=\alpha_{3}=0, \alpha_{4}=1 \\
\beta_{0}=t-\frac{14}{45} h-\frac{17}{96 h^{3}} t^{4}+\frac{1}{40 h^{4}} t^{5}-\frac{1}{720 h^{5}} t^{6} \\
\beta_{1}=-\frac{64}{45} h+\frac{5}{2 h} t^{2}-\frac{77}{36 h^{2}} t^{3}+\frac{71}{96 h^{3}} t^{4}-\frac{7}{60 h^{4}} t^{5}+\frac{1}{144 h^{5}} t^{6} \\
\beta_{2}=-\frac{8}{15} h-\frac{5}{2 h} t^{2}-\frac{59}{48 h^{3}} t^{4}-\frac{1}{72 h^{5}} t^{6}+\frac{13}{60 h^{4}} t^{5}+\frac{107}{36 h^{2}} t^{3}
\end{gathered}
$$




$$
\begin{aligned}
& \beta_{3}=-\frac{64}{45} h+\frac{5}{3 h} t^{2}-\frac{13}{6 h^{2}} t^{3}+\frac{49}{48 h^{3}} t^{4}-\frac{1}{5 h^{4}} t^{5}+\frac{1}{72 h^{5}} t^{6} \\
& \beta_{4}=\frac{11}{120 h^{4}} t^{5}-\frac{14}{45} h+\frac{61}{72 h^{2}} t^{3}-\frac{1}{144 h^{5}} t^{6}-\frac{41}{96 h^{3}} t^{4}-\frac{5}{8 h} t^{2} \\
& \beta_{5}=\frac{1}{10 h} t^{2}-\frac{5}{36 h^{2}} t^{3}+\frac{7}{96 h^{3}} t^{4}-\frac{1}{60 h^{4}} t^{5}+\frac{1}{720 h^{5}} t^{6}
\end{aligned}
$$

Evaluating (6) with coefficients (7) at $x_{n}, x_{n+1}, x_{n+2}, x_{n+3}$ and $x_{n+5}$ with $t=\left(x-x_{n}\right)$ the following discrete schemes is respectively obtained as;

$$
\begin{gathered}
y_{n}=y_{n+4}-\frac{14}{45} h f_{n}-\frac{64}{45} h f_{n+1}-\frac{8}{15} h f_{n+2}-\frac{64}{45} h f_{n+3}-\frac{14}{45} h f_{n+4} \\
y_{n+1}=y_{n+4}+\frac{3}{160} h f_{n}-\frac{69}{160} h f_{n+1}-\frac{87}{80} h f_{n+2}-\frac{87}{80} h f_{n+3}-\frac{69}{160} h f_{n+4}+\frac{3}{160} h f_{n+5} \\
y_{n+2}=y_{n+4}+\frac{1}{90} h f_{n+1}-\frac{17}{45} h f_{n+2}-\frac{19}{15} h f_{n+3}-\frac{17}{45} h f_{n+4}+\frac{1}{90} h f_{n+5} \\
y_{n+3}=y_{n+4}+\frac{11}{1440} h f_{n}-\frac{77}{1440} h f_{n+1}+\frac{43}{240} h f_{n+2}-\frac{511}{720} h f_{n+3}-\frac{637}{1440} h f_{n+4}+\frac{3}{160} h f_{n+5} \\
y_{n+5}=y_{n+4}+\frac{3}{160} h f_{n}-\frac{173}{1440} h f_{n+1}+\frac{241}{720} h f_{n+2}-\frac{133}{240} h f_{n+3}+\frac{1427}{1440} h f_{n+4}+\frac{95}{288} h f_{n+5}
\end{gathered}
$$

\subsection{Five Step Method with Three Off-Grid Points}

Interpolating (2) at $x_{n+4}$ and collocating (3) at $x_{n}, x_{n+1}, x_{n+2}, x_{n+3}, x_{n+4}, x_{n+\frac{13}{3}}, x_{n+\frac{9}{2}}, x_{n+\frac{14}{3}}$ and $x_{n+5}$ gives a system of nonlinear equations of the form

$$
\mathrm{AX}=\mathrm{B}
$$

where

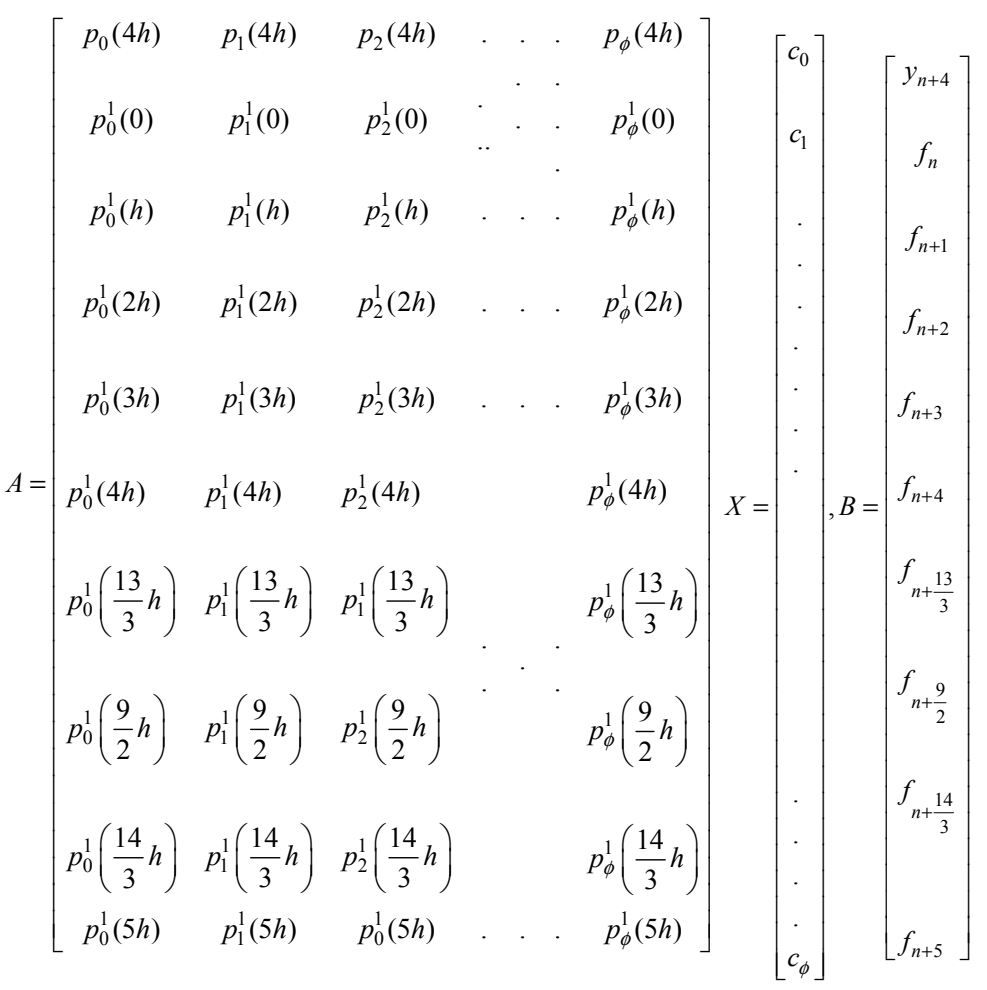

Solving for the $C_{i}{ }^{\prime} s$ using inverse of a matrix method and substituting in (2) gives the continuous formulation 


$$
y(x)=\sum_{j=0}^{k-1} \alpha_{j}(x) y_{n+j}+h \sum_{j=0}^{k} \beta_{j}(x) f_{n+j}+h \sum_{\omega_{i}} \beta_{\omega_{i}}(x) f_{n+\omega_{i}}
$$

where

$$
\begin{aligned}
& k=5, \omega_{1}=\frac{13}{3}, \omega_{2}=\frac{9}{2}, \omega_{3}=\frac{14}{3}, \alpha_{0}=\alpha_{1}=\alpha_{2}=\alpha_{3}=0 \text { and } \alpha_{4}=1 \\
& \beta_{0}=t-\frac{351518}{1289925} h-\frac{48331}{32760 h} t^{2}+\frac{87149}{73710 h^{2}} t^{3}-\frac{16811}{29120 h^{3}} t^{4}+\frac{88541}{491400 h^{4}} t^{5}-\frac{7123}{196560 h^{5}} t^{6}+\frac{1567}{343980 h^{6}} t^{7}-\frac{19}{58240 h^{7}} t^{8}+\frac{1}{98280 h^{6}} t^{9} \\
& \beta_{1}=\frac{3}{896 h^{7}} t^{8}-\frac{31951}{4620 h^{2}} t^{3}-\frac{1}{9240 h^{8}} t^{9}-\frac{212552}{121275} h+\frac{15689}{3696 h^{3}} t^{4}+\frac{117}{22 h} t^{2}-\frac{140117}{92400 h^{4}} t^{5}+\frac{7393}{22176 h^{5}} t^{6}-\frac{5773}{129360 h^{6}} t^{7} \\
& \beta_{2}=\frac{10016}{11025} h-\frac{117}{8 h} t^{2}+\frac{40141}{1680 h^{2}} t^{3}-\frac{228173}{13440 h^{3}} t^{4}+\frac{56431}{8400 h^{4}} t^{5}-\frac{3211}{2016 h^{5}} t^{6}+\frac{2657}{11760 h^{6}} t^{7}-\frac{159}{8960 h^{7}} t^{8}+\frac{1}{1680 h^{8}} t^{9} \\
& \beta_{3}=\frac{91}{2 h} t^{2}-\frac{31672}{4725} h-\frac{42871}{540 h^{2}} t^{3}+\frac{14603}{240 h^{3}} t^{4}-\frac{92887}{3600 h^{4}} t^{5}-\frac{4891}{5040 h^{6}} t^{7}+\frac{1871}{288 h^{5}} t^{6}+\frac{51}{640 h^{7}} t^{8}-\frac{1}{360 h^{8}} t^{9} \\
& \beta_{4}=\frac{19454}{315} h-\frac{4095}{8 h} t^{2}+\frac{11059}{12 h^{2}} t^{3}-\frac{141121}{192 h^{3}} t^{4}+\frac{39097}{120 h^{4}} t^{5}-\frac{12361}{144 h^{5}} t^{6}+\frac{563}{42 h^{6}} t^{7}-\frac{147}{128 h^{7}} t^{8}+\frac{1}{24 h^{8}} t^{9} \\
& \beta_{\frac{13}{3}}=\frac{59049}{26 h} t^{2}-\frac{4391496}{15925} h-\frac{7494849}{1820 h^{2}} t^{3}+\frac{4826709}{1456 h^{3}} t^{4}+\frac{1154007}{2912 h^{5}} t^{6}-\frac{3195207}{50960 h^{6}} t^{7}-\frac{729}{3640 h^{8}} t^{9}+\frac{63423}{11648 h^{7}} t^{8}-\frac{54082323}{36400 h^{4}} t^{5} \\
& \beta_{\frac{9}{2}}=\frac{35618816}{99225} h-\frac{26624}{9 h} t^{2}+\frac{15254528}{2835 h^{2}} t^{3}-\frac{456704}{105 h^{3}} t^{4}+\frac{9257216}{4725 h^{4}} t^{5}-\frac{99328}{189 h^{5}} t^{6}+\frac{553472}{6615 h^{6}} t^{7}-\frac{256}{35 h^{7}} t^{8}+\frac{256}{945 h^{8}} t^{9} \\
& \beta_{\frac{14}{3}}=\frac{767637}{616 h} t^{2}-\frac{2035368}{13475} h-\frac{14003361}{6160 h^{2}} t^{3}+\frac{90907029}{49280 h^{3}} t^{4}-\frac{25712559}{30800 h^{4}} t^{5}+\frac{554769}{2464 h^{5}} t^{6}-\frac{1554957}{43120 h^{6}} t^{7}+\frac{28431}{8960 h^{7}} t^{8}-\frac{729}{6160 h^{8}} t^{9} \\
& \beta_{5}=\frac{15592}{1575} h-\frac{819}{10 h} t^{2}+\frac{9011}{60 h^{2}} t^{3}-\frac{29453}{240 h^{3}} t^{4}+\frac{67217}{1200 h^{4}} t^{5}-\frac{21973}{1440 h^{5}} t^{6}+\frac{4153}{1680 h^{6}} t^{7}-\frac{141}{640 h^{7}} t^{8}+\frac{1}{120 h^{8}} t^{9}
\end{aligned}
$$

Evaluating (10) with coefficients (11) at $x_{n}, x_{n+1}, x_{n+2}, x_{n+3}, x_{n+\frac{13}{3}}, x_{n+\frac{9}{2}}, x_{n+\frac{14}{3}}$ and $x_{n+5}$ with $t=\left(x-x_{n}\right)$ the following discrete schemes are respectively obtained as;

$$
\begin{gathered}
y_{n}=y_{n+4}-\frac{351518}{1289925} h f_{n}-\frac{212552}{121275} h f_{n+1}+\frac{10016}{11025} h f_{n+2}-\frac{31672}{4725} h f_{n+3}+\frac{19454}{315} h f_{n+4}+\frac{15592}{1575} h f_{n+5} \\
+\frac{35618816}{99225} h f_{n+\frac{9}{2}}-\frac{4391496}{15925} h f_{n+\frac{13}{3}}-\frac{2035368}{13475} h f_{n+\frac{14}{3}} \\
y_{n+1}=\frac{32951}{6115200} h f_{n}+y_{n+4}-\frac{568893}{1724800} h f_{n+1}-\frac{459807}{313600} h f_{n+2}+\frac{1189}{22400} h f_{n+3}-\frac{50499}{4480} h f_{n+4}-\frac{34107}{22400} h f_{n+5} \\
-\frac{212224}{3675} h f_{n+\frac{9}{2}}+\frac{92569149}{2038400} h f_{n+\frac{13}{3}}+\frac{82333989}{3449600} h f_{n+\frac{14}{3}} \\
y_{n+2}=y_{n+4}-\frac{247}{396900} h f_{n}+\frac{43}{3675} h f_{n+1}-\frac{3509}{9800} h f_{n+2}-\frac{6701}{4725} h f_{n+3}+\frac{871}{420} h f_{n+4}+\frac{69}{175} h f_{n+5}+\frac{1466368}{99225} h f_{n+\frac{9}{2}} \\
-\frac{13851}{1225} h f_{n+\frac{13}{3}}-\frac{60507}{9800} h f_{n+\frac{14}{3}} \\
\quad-\frac{745216}{99225} h f_{n+\frac{9}{2}}+\frac{13557213}{2038400} h f_{n+\frac{13}{3}}+\frac{9737253}{3449600} h f_{n+\frac{14}{3}} \\
y_{n+3}=\frac{14669}{165110400} h f_{n}+y_{n+4}-\frac{20869}{15523200} h f_{n+1}+\frac{36329}{2822400} h f_{n+2}-\frac{202169}{604800} h f_{n+3}-\frac{100187}{40320} h f_{n+4}-\frac{31411}{201600} h f_{n+5} \\
y_{n+\frac{13}{3}} \frac{690797}{1083289334400} h f_{n}+y_{n+4}-\frac{910757}{101847715200} h f_{n+1}+\frac{1336457}{18517766400} h f_{n+2}-\frac{2512217}{3968092800} h f_{n+3}+\frac{31844549}{264539520} h f_{n+4}
\end{gathered}
$$




$$
\begin{aligned}
& -\frac{3364243}{1322697600} h f_{n+5}-\frac{134364928}{651015225} h f_{n+\frac{9}{2}}+\frac{19955023}{55036800} h f_{n+\frac{13}{3}}+\frac{5577703}{93139200} h f_{n+\frac{14}{3}} \\
& y_{n+\frac{9}{2}}=\frac{24863}{42268262400} h f_{n}+y_{n+4}-\frac{32833}{3973939200} h f_{n+1}+\frac{48323}{722534400} h f_{n+2}-\frac{91493}{154828800} h f_{n+3}+\frac{1220071}{10321920} h f_{n+4} \\
& -\frac{115447}{51609600} h f_{n+5}-\frac{21389}{198450} h f_{n+\frac{9}{2}}+\frac{231041241}{521830400} h f_{n+\frac{13}{3}}+\frac{43797591}{883097600} h f_{n+\frac{14}{3}} \\
& y_{n+\frac{14}{3}}=\frac{22037}{33852791700} h f_{n}+y_{n+4}-\frac{7253}{795685275} h f_{n+1}+\frac{42467}{578680200} h f_{n+2}-\frac{19853}{31000725} h f_{n+3}+\frac{993749}{8266860} h f_{n+4} \\
& -\frac{29107}{10333575} h f_{n+5}+\frac{7757824}{651015225} h f_{n+\frac{9}{2}}+\frac{180667}{429975} h f_{n+\frac{13}{3}}+\frac{342733}{2910600} h f_{n+\frac{14}{3}} \\
& y_{n+5}=y_{n+4}-\frac{179}{165110400} h f_{n}+\frac{73}{5174400} h f_{n+1}-\frac{31}{313600} h f_{n+2}+\frac{359}{604800} h f_{n+3}+\frac{1079}{13440} h f_{n+4}+\frac{1989}{22400} h f_{n+5}-\frac{61184}{99225} h f_{n+\frac{9}{2}}+ \\
& \frac{1496637}{2038400} h f_{n+\frac{13}{3}}+\frac{2458917}{3449600} h f_{n+\frac{14}{3}}
\end{aligned}
$$

\section{Analysis of the Method}

In this section the error constant, order, consistency, zero stability, convergent and region of absolute stability of the schemes generated are discussed.

\subsection{Order and Error Constant}

Expanding (8) and (12) in Taylor's series and collecting like terms in powers of $h$, the order and error constant are respectively obtained as follows;

Table 1. Order and Error Constants of the Discrete Schemes of the Block Method (8).

\begin{tabular}{lll}
\hline Scheme & Order & Error constant \\
\hline$y_{n+1}$ & 6 & $C_{7}=-5.8035714 \times 10^{-3}$ \\
$y_{n+2}$ & 6 & $C_{7}=-1.3227513 \times 10^{-3}$ \\
$y_{n+3}$ & 6 & $C_{7}=-4.4808201 \times 10^{-3}$ \\
$y_{n+4}$ & 6 & $C_{7}=8.46560850 \times 10^{-3}$ \\
$y_{n+5}$ & 6 & $C_{7}=-1.4269180 \times 10^{-2}$ \\
\hline
\end{tabular}

Table 2. Order and Error Constants of the Discrete Schemes of the Hybrid Block Method (12).

\begin{tabular}{lll}
\hline Scheme & Order & Error constant \\
\hline$y_{n+1}$ & 9 & $C_{10}=4.9057933 \times 10^{-4}$ \\
$y_{n+2}$ & 9 & $C_{10}=-4.255200 \times 10^{-5}$ \\
$y_{n+3}$ & 9 & $C_{10}=9.04215420 \times 10^{-6}$ \\
$y_{n+4}$ & 9 & $C_{10}=-84.29153100000$ \\
$y_{n+\frac{13}{3}}$ & 9 & $C_{10}=-0.643365680000$ \\
$y_{n+\frac{9}{2}}$ & 9 & $C_{10}=7.29157760 \times 10^{-8}$ \\
$y_{n+\frac{14}{3}}$ & 9 & $C_{10}=8.15819800 \times 10^{-8}$ \\
$y_{n+5}$ & 9 & $C_{10}=-1.7278002 \times 10^{-7}$ \\
\hline
\end{tabular}

Hence the block methods are of order $\check{\rho}=6,9$ and error constant of $\check{C}_{7}, \check{C}_{10}$ respectively

\subsection{Consistency}

Following [8] and [6], the block methods are said to be consistent if they satisfy the following conditions:

(i) the order $\check{p} \geq 1$ (ii) $\sum_{j=0}^{k} \check{\alpha}_{j}=0$

(iii) $\rho$ (1) $=\rho^{\prime}(1)$

(iv) $\rho^{\prime \prime}(1)=2 ! \sigma(1)$

Where $\rho(r)$ and $\sigma(r)$ are the first and second characteristics polynomials of the block method. According to [8] and [6], condition (i) above is a sufficient condition for the block methods to be consistent. Hence the block methods are consistent since $\breve{p}=6,9>1$.

\subsection{Zero Stability}

The block methods are said to be zero stable if the roots $z_{r} ; r=1, \ldots, n$ of the first characteristic polynomial $p(z)$, defined by

$$
p(z)=\operatorname{det}|z Q-T|
$$

satisfies $\left|z_{r}\right| \leq 1$ and every root with $\left|z_{r}\right|=1$ has multiplicity not exceeding two in the limit as $h \rightarrow 0$. Calculations from all available information revealed that the block methods (8) and (12) have the following roots respectively.

$$
z^{4}(z-1)=0 \text { and } z^{8}(z-1)=0
$$

Hence the block methods are zero stable, since all roots with modulus one do not have multiplicity exceeding the order of the differential equation in the limit as $h \rightarrow 0$.

\subsection{Convergence}

According to [7], we can safely assert the convergence of the block methods (8) and (12)

\subsection{Region of Absolute Stability}

Reformulating the block methods (8) and (12) as a General Linear Methods (GLM) containing a partition of matrices A, Band $\mathrm{C}$ and then substituting into the stability polynomial $r(A-C z)-B$. Using a MATLAB code based on the idea of Newton's iteration, the regions of absolute stability of the block methods are respectively shown below. 


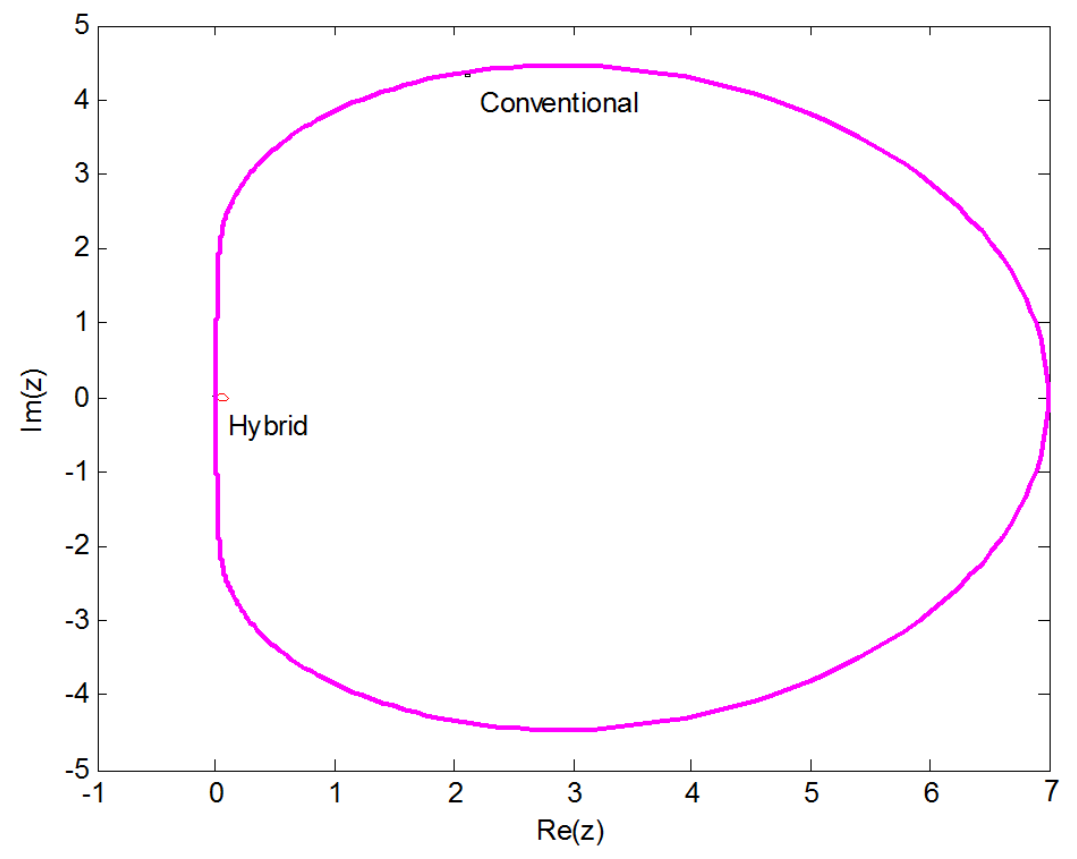

Figure 1. The regions of absolute stability for methods (8) and (12).

\section{Numerical Illustrations}

The following numerical experiments are performed with the aid of MAPLE 18 software package in order to further affirm the earlier established convergence of the methods.

Example 1

The ordinary differential equation

$$
y^{\prime}=5 y, 0 \leq x \leq 1, y(0)=1, h=0.01
$$

The exact solution is $y(x)=e^{5 x}$. ([12]) Example 2
The ordinary differential equation

$$
y^{\prime}=x-y, 0 \leq x \leq 1, y(0)=0, h=0.1
$$

The exact solution is $y(x)=x+e^{-x}-1$. ([10]). Example 3

The ordinary differential equation

$$
y^{\prime}=y-x^{2}+1,0 \leq x \leq 2, y(0)=0.5, h=0.2
$$

\begin{tabular}{|c|c|c|c|c|c|}
\hline$x$ & Exact Solution & Result of Method (8) & Absolute error of (8) & Result of Method (12) & Absolute error of (12) \\
\hline 0.01 & 1.05127109637602 & 1.05127109638904 & $1.302 \times 10^{-11}$ & 1.05127109637607 & $5.0 \times 10^{-14}$ \\
\hline 0.02 & 1.10517091807565 & 1.10517091808526 & $9.610 \times 10^{-12}$ & 1.10517091807569 & $4.0 \times 10^{-14}$ \\
\hline 0.03 & 1.16183424272828 & 1.16183424274126 & $1.298 \times 10^{-11}$ & 1.16183424272835 & $7.0 \times 10^{-14}$ \\
\hline 0.04 & 1.22140275816017 & 1.22140275816975 & $9.580 \times 10^{-12}$ & 1.22140275816017 & 0.00000000 \\
\hline 0.05 & 1.28402541668774 & 1.28402541671072 & $2.298 \times 10^{-11}$ & 1.28402541668780 & $6.0 \times 10^{-14}$ \\
\hline 0.06 & 1.34985880757600 & 1.34985880761687 & $4.087 \times 10^{-11}$ & 1.34985880757611 & $1.1 \times 10^{-13}$ \\
\hline 0.07 & 1.41906754859326 & 1.41906754863098 & $3.772 \times 10^{-11}$ & 1.41906754859338 & $1.2 \times 10^{-13}$ \\
\hline 0.08 & 1.49182469764127 & 1.49182469768462 & $4.335 \times 10^{-11}$ & 1.49182469764142 & $1.5 \times 10^{-13}$ \\
\hline 0.09 & 1.56831218549017 & 1.56831218553052 & $4.035 \times 10^{-11}$ & 1.56831218549031 & $1.4 \times 10^{-13}$ \\
\hline 0.10 & 1.64872127070013 & 1.64872127075914 & $5.901 \times 10^{-11}$ & 1.64872127070028 & $1.5 \times 10^{-13}$ \\
\hline
\end{tabular}

The exact solution is $y(x)=(x+1)^{2}+0.5 e^{x}$, ([13])

Table 3. Absolute Error Values for Example 1 of Methods (8) and (12).

\begin{tabular}{|c|c|c|c|c|c|}
\hline$x$ & Exact Solution & Result of Method (8) & Absolute error of (8) & Result of Method (12) & Absolute error of (12) \\
\hline 0.1 & 0.004837418035960 & 0.0048374169836170 & $1.05 \times 10^{-9}$ & 0.0048374180358260 & $1.34 \times 10^{-13}$ \\
\hline 0.2 & 0.018730753077982 & 0.0187307524576758 & $6.20 \times 10^{-10}$ & 0.0187307530778861 & $9.59 \times 10^{-14}$ \\
\hline 0.3 & 0.040818220681718 & 0.0408182198857461 & $7.96 \times 10^{-10}$ & 0.0408182206816284 & $8.96 \times 10^{-14}$ \\
\hline 0.4 & 0.070320046035639 & 0.0703200456495184 & $3.86 \times 10^{-10}$ & 0.0703200460355589 & $8.01 \times 10^{-14}$ \\
\hline 0.5 & 0.106530659712633 & 0.106530658294696 & $1.42 \times 10^{-9}$ & 0.106530659712561 & $7.20 \times 10^{-14}$ \\
\hline 0.6 & 0.148811636094026 & 0.148811634172747 & $1.92 \times 10^{-9}$ & 0.148811636093972 & $5.40 \times 10^{-14}$ \\
\hline 0.7 & 0.196585303791410 & 0.196585302254268 & $1.54 \times 10^{-9}$ & 0.196585303791371 & $3.90 \times 10^{-14}$ \\
\hline 0.8 & 0.249328964117222 & 0.249328962584008 & $1.53 \times 10^{-9}$ & 0.249328964117176 & $4.60 \times 10^{-14}$ \\
\hline 0.9 & 0.306569659740599 & 0.306569658555933 & $1.85 \times 10^{-9}$ & 0.306569659740559 & $4.00 \times 10^{-14}$ \\
\hline 1.0 & 0.367879441171442 & 0.367879439451397 & $1.72 \times 10^{-9}$ & 0.367879441171405 & $3.70 \times 10^{-14}$ \\
\hline
\end{tabular}

Table 4. Absolute Error Values for Example 2 of Methods (8) and (12). 
Table 5. Absolute Error Values for Example 3 of Methods (8) and (12).

\begin{tabular}{|c|c|c|c|c|c|}
\hline$x$ & Exact Solution & Result of Method (8) & Absolute error of (8) & Result of Method (12) & Absolute error of (12) \\
\hline 0.2 & 0.829298620919915 & 0.829298449813542 & $1.71 \times 10^{-7}$ & 0.829298621130485 & $2.11 \times 10^{-10}$ \\
\hline 0.4 & 1.21408765117936 & 1.21408749545914 & $1.56 \times 10^{-7}$ & 1.21408765140944 & $2.30 \times 10^{-10}$ \\
\hline 0.6 & 1.64894059980474 & 1.64894037231163 & $2.27 \times 10^{-7}$ & 1.64894060009137 & $2.87 \times 10^{-10}$ \\
\hline 0.8 & 2.12722953575376 & 2.12722931045984 & $2.25 \times 10^{-7}$ & 2.12722953610287 & $3.49 \times 10^{-10}$ \\
\hline 1.0 & 2.64085908577048 & 2.64085864464325 & $4.41 \times 10^{-7}$ & 2.64085908619686 & $4.26 \times 10^{-10}$ \\
\hline 1.2 & 3.17994153863172 & 3.17994053472221 & $1.00 \times 10^{-6}$ & 3.17994153972539 & $1.09 \times 10^{-10}$ \\
\hline 1.4 & 3.73240001657766 & 3.73239893520156 & $1.08 \times 10^{-6}$ & 3.73240001783966 & $1.26 \times 10^{-9}$ \\
\hline 1.6 & 4.28348378780244 & 4.28348236562564 & $1.42 \times 10^{-6}$ & 4.28348378935910 & $1.56 \times 10^{-9}$ \\
\hline 1.8 & 4.81517626779352 & 4.81517467363426 & $1.59 \times 10^{-6}$ & 4.81517626969216 & $1.90 \times 10^{-9}$ \\
\hline 2.0 & 5.30547195053468 & 5.30546955231802 & $2.40 \times 10^{-6}$ & 5.30547195285364 & $2.32 \times 10^{-9}$ \\
\hline
\end{tabular}

\section{Discussion of Result}

Two different methods for solving first order initial value problems of ordinary differential equations have been proposed in this work, the conventional or standard method and the hybrid method with the hybrid having more advantages over the conventional method the hybrid higher order and accuracy. The methods does not require developing separate predictors to implement this makes it simple and attractive for solving initial value problems of ordinary differential equations. A careful observation of Tables 1, 2 and 3 showed that both standard and hybrid methods performed well as their absolute error values are convergent, hence affirming the earlier established convergence of the methods. The hybrid method is useful as it reduces the step number of a method and still remains zero stable; in addition, the absolute error values presented in Tables 3, 4 and 5 indicated that the hybrid method performed better than the standard method when applied to stiff and non-stiff differential equations respectively. The results also revealed that the hybrid method converges faster than the standard method, since it has minimum absolute error values.

\section{Conclusion}

Justifying from the numerical calculations, it has been observed that the hybrid method performed well than the conventional method, it has also been established from the calculations that the hybrid method has high order and relatively small error constants than the conventional method. Finally, it has been established that hybrid methods gives better results than the standard methods when applied to either stiff or non-stiff initial value problems.

\section{References}

[1] Adee, S. O., Onumanyi, P., Sirisena, U. W. andYahaya, Y. A. (2005) Note on Starting the Numerov Method More Accurately by a Hybrid Formula of Order Four for Initial Value Problems. Journal of Computational and Applied Mathematics, 175: 369-373.
[2] Ademiluyi, R. A. (1987) New Hybrid Methods for Systems of Stiff Equations, Benin City, Nigeria: PhD Thesis, University of Benin.

[3] Akinfenwa, O. A., Jator, S. N. and Yao, N. M. (2011) Linear Multistep Hybrid Methods with Continuous Coefficients for Solving Stiff Ordinary Differential Equations, Journal of Modern Mathematics and Statistics, 5(2): 47-57.

[4] Anake, T. A. (2011). Continuous Implicit Hybrid One-Step Methods for the Solution of Initial Value Problems of Second Order Ordinary Differential Equations. Ogun State, Nigeria. $\mathrm{PhD}$ Thesis, Covenant University, Ota.

[5] Awari, Y. S., Abada, A. A., Emma, P. M. and Kamoh, N. M. (2013) Application of Two Step Continuous Hybrid Butcher's Method in Block Form for the Solution of First Order Initial Value Problems. Natural and Applied Sciences, 4(4): 209-218.

[6] Fatunla, S. O. (1988) Numerical Methods for Initial Value Problems for Ordinary Differential Equations USA, Academy press, Boston 295.

[7] Henrici, P. (1962) Discrete Variable Methods for ODE`s, New York, USA, John Wiley and Sons, (1962).

[8] Lambert, J. D. (1991) Numerical Methods for Ordinary Differential Equations, New York: John Wiley and Sons pp 293.

[9] Mohammed, U. and Adeniyi, R. B (2014) A Three Step Implicit Hybrid Linear Multistep Method for the Solution of Third Order Ordinary Differential Equations. General Mathematics Notes, 25(1): 62-74.

[10] Odejide, S. A. and Adenira, A. O. (2012) A Hybrid Linear Collocation Multistep Scheme For Solving First Order Initial Value Problems. Journal of the Nigerian Mathematical Society 31: 229-241.

[11] Serisina, U. W., Kumleng, G. M. and Yahaya, Y. A. (2004) A New Butcher Type Two-Step Block Hybrid Multistep Method for Accurate and Efficient Parallel Solution of Ordinary Differential Equations, Abacus Mathematics Series. 31: 1-7.

[12] Yakusak, N. S., Emmanuel, S. and Ogunniran, M. O. (2015) Uniform Order Legendre Approach for Continuous Hybrid Block Methods for the Solution of First Order Ordinary Differential Equations. IOSR Journal of Mathematics, 11(1): 09-14.

[13] Zill, D. G. and Warren, W. S. (2013) Differential Equations with Boundary-Value Problems, Cengage Learning: Eight edition books/Cole. 